\title{
Statistical Interparticle Potential of an Ideal Gas of Non-Abelian Anyons
}

\author{
Francesco Mancarella, ${ }^{1}$ Andrea Trombettoni,${ }^{2,1}$ and Giuseppe Mussardo ${ }^{1,3}$ \\ ${ }^{1}$ SISSA and INFN, Sezione di Trieste, \\ via Bonomea 265, I-34136 Trieste, Italy \\ ${ }^{2}$ CNR-IOM DEMOCRITOS Simulation Center, \\ Via Bonomea 265, I-34136 Trieste, Italy \\ ${ }^{3}$ International Centre for Theoretical Physics (ICTP), \\ Strada Costiera 11, I-34151, Trieste, Italy
}

We determine and study the statistical interparticle potential of an ideal system of non-Abelian Chern-Simons (NACS) particles, comparing our results with the corresponding results of an ideal gas of Abelian anyons. In the Abelian case, the statistical potential depends on the statistical parameter $\alpha$ and it has a "quasi-bosonic" behaviour for $0<\alpha<1 / 2$ (non-monotonic with a minimum) and a "quasi-fermionic" behaviour for $1 / 2<\alpha<1$ (monotonically decreasing without a minimum). In the non-Abelian case the behavior of the statistical potential depends on the ChernSimons coupling and the isospin quantum number: as a function of these two parameters, a phase diagram with quasi-bosonic, quasi-fermionic and bosonic-like regions is obtained and investigated. Finally, using the obtained expression for the statistical potential, we compute the second virial coefficient of the NACS gas, which correctly reproduces the results available in literature.

Keywords: Fractional statistics; Anyon thermodynamics; Effective statistical potential; Chern-Simons theory

PACS number: 05.30.Pr

Contact information: Francesco Mancarella (corresp. author): mancarel@sissa.it 


\section{INTRODUCTION}

A key property of the statistics of quantum systems in two space dimensions is provided by the possibility to display intermediate fractional statistics interpolating between bosons and fermions: the properties of anyons, the two-dimensional identical particles obeying fractional braiding statistics and carrying fractional charge, have been the subject of an intense and continuing interest [1 5]. Both Abelian and non-Abelian anyons (associated to respectively one-dimensional and irreducible higher-dimensional representations of the braid group) have been extensively studied both for their intrinsic interest and their connection with quantum Hall systems [5-7]. In particular there is an increasing interest in the investigation of the properties of non-Abelian anyons for their application to topologically fault-tolerant quantum information processing [5].

The ideal gas of anyons is also interesting for the phenomenon of statistical transmutation [2 4], i.e. the fact that one can treat non-interacting anyons as interacting bosons or fermions. The idea behind statistical transmutation is that one can alternatively consider the system as made of interacting particles with canonical statistics or of non-interacting particles, but obeying non-canonical statistics. This makes in general difficult the study of

the equilibrium thermodynamical properties of the ideal anyon gases [4] and therefore any result that gives even qualitative informations on such ideal gases is valuable.

Dating back to the seminal work by Uhlenbeck and Gropper [8], a standard way to characterize the effects of the quantum statistics on the properties of ideal gases is provided by the determination of the so-called statistical potential. As detailed in textbooks [9, 10], one can show that the partition function $(\mathrm{PF})$ of a gas of particles approaches - for sufficiently high temperatures - the PF of the classical gas (with the correct Boltzmann counting). When this computation is done for an ideal quantum gas (under the condition that the thermal wavelength is much smaller than the interparticle distance) one finds that the quantum $\mathrm{PF}$ becomes the PF of the classical ideal gas. Evaluating the first quantum correction, one can appreciate that the quantum PF can be formally written as the PF a classical gas in which a fictitious two-body interaction term (the statistical potential) is added [9, 10]. The statistical potential gives a simple characterization of the effects of the quantum statistics of the ideal gases: the statistical potential is "attractive" for bosons and "repulsive" for fermions, and it is respectively monotonically increasing (decreasing) for bosons (fermions). 
Another important result is that a suitable integral of the statistical interparticle potential gives the second coefficient of the virial expansion [9, 10].

The statistical potential of a gas of ideal Abelian anyons has been studied in [11 13] and it depends on the statistical parameter $\alpha$ (we remind that $\alpha=0$ and $\alpha=1$ corresponds respectively to free two-dimensional spinless bosons and fermions, while $\alpha=1 / 2$ corresponds to semions [4]). It is found that for $1 / 2 \leq \alpha \leq 1$ the statistical potential $v_{\alpha}(r)$ is monotonically decreasing, while for $0<\alpha<1 / 2$ it has a a minimum at a finite value of $r$ and it is increasing for larger values of $r$ (while for $\alpha=0$ is monotonically increasing). We can refer to these behaviors respectively as "quasi-fermionic" $(1 / 2 \leq \alpha<1)$ and "quasi-bosonic" $(0<\alpha<1 / 2)$. The purpose of the present paper is to compute the statistical potential of the ideal gas of non-Abelian anyons: we find that the behaviour of the statistical potential depends on the Chern-Simons coupling and the isospin quantum number. As a function of these two parameters, quasi-bosonic and quasi-fermionic regions emerge, and they are part of a phase diagram which will be presented below.

The plan of the paper is the following: in Section II we recall the steps which lead to the computation of the statistics potential $v_{\alpha}$ of an ideal gas of Abelian anyons with statistical parameter $\alpha$. Since it is possible to show that the statistical potential for the non-Abelian gas may be written in terms of sums of Abelian statistical potentials (having different statistical parameters depending on the projection of the isospin quantum number), we provide in Section II a detailed study of $v_{\alpha}$ : we present a compact and useful integral representation for it, showing that it can be written in terms of bivariate Lommel functions [14]. Furthermore, using the Sumudu transform of the statistical potential [15], we also give a closed expression of $v_{\alpha}(r)$ in terms of the inverse Laplace transform of an algebraic function of $r$ and $\alpha$. Using this result we are able to give a simple expression for the statistical potential of the ideal gas of semions, and we show that for a general value $\alpha$ it is possible to retrieve the well-known result for the second virial coefficient of an ideal anyon gas found in [16] (with an hard-core boundary condition for the two-body wavefunction at zero distance). For the sake of comparison with the non-Abelian case that follows, the limit behaviors both at small and large distance of the statistics potentials are presented. In Section III we introduce the non-Abelian Chern-Simons (NACS) model studied in the rest of the paper: we compute the statistics potential (within the hard-core boundary condition frame) as a function of the Chern-Simons coupling $\kappa$ and the isospin quantum number $l$ 
and we build a phase diagram summarizing the behavior of the statistical potential in terms of $\kappa$ and $l$. We then show that the second virial coefficient, previously studied in [17-20], is correctly retrieved. The asymptotic expressions for the small and large distance of the statistics potentials are also given. Finally, our conclusions are drawn in Section IV, while supplementary material is presented in the Appendices.

\section{STATISTICAL POTENTIAL FOR ABELIAN ANYONS}

In this Section we introduce the model for an ideal gas of Abelian anyons, and we then derive its statistical potential $v_{\alpha}(r)$ as a function of the statistical parameter $\alpha$, obtaining the expression for $v_{\alpha}(r)$ given in [11 13], and also providing an explicit formula for the semions (half-integer values of $\alpha$ ). The results for $v_{\alpha}(r)$ and the asymptotic expressions for small and large distance will be used in the next Section, where the statistical inter-particle potential of an ideal gas of non-Abelian anyons is derived and studied.

Abelian anyons admit a concrete representation by the flux-charge composite model [4], and the statistics of these objects can be understood in terms of Aharonov-Bohm type interference [21, 22]. The Hamiltonian for the quantum dynamics of an ideal system of anyons reads [3, 4]

$$
H_{N}=\sum_{n=1}^{N} \frac{1}{2 M}\left(\vec{p}_{n}-\alpha \vec{a}_{n}\right)^{2},
$$

where $\vec{p}_{n}$ is the momentum of the $n$-th particle $(n=1, \cdots, N)$. Similarly we will denote the position of the $n$-th particle by $\vec{r}_{n} \equiv\left(x_{n}^{1}, x_{n}^{2}\right)$. In Eq.(1) $\alpha$ is the statistical parameter: notice that the physical quantities, e.g. the virial coefficients, are periodic with period 2 [4]: the bosonic points are defined by $\alpha=2 j$ and the fermionic ones by $\alpha=2 j+1, j$ integer. For this reason we will consider in the following $\alpha \in[0,2]$.

In Eq.(11) $\vec{a}_{n}$ is the vector potential carrying the flux attached to the bosons: indeed, the Hamiltonian (11) is written in the so-called bosonic representation, i.e. it is an Hamiltonian for identical bosons, and therefore acting on the subspace of wavefunctions which are symmetric with respect to the exchange of particles. The explicit expression for $\vec{a}_{n} \equiv\left(a_{n}^{1}, a_{n}^{2}\right)$ is

$$
a_{n}^{i}=\hbar \epsilon^{i j} \sum_{m(\neq n)} \frac{x_{n}^{j}-x_{m}^{j}}{\left|\vec{r}_{n}-\vec{r}_{m}\right|^{2}} .
$$

where $\epsilon^{i j}$ is the totally antisymmetric tensor $(i, j=1,2)$. 
Let's recall that this model of Abelian anyons also admits a field-theoretic description: in fact (non-relativistic) anyons can be described by bosonic Schrödinger fields $\psi$ and $\psi^{\dagger}$ coupled to a Chern-Simons gauge field $a_{\mu}$ living in (2+1)-D [23, 24] (then $\mu=0,1,2$ ). The Lagrangian density of such a system reads

$$
\mathcal{L}=\frac{c}{2} \epsilon^{\mu \nu \lambda} a_{\mu} \partial_{\nu} a_{\lambda}+\psi^{\dagger}\left(i D_{t}+\frac{1}{2 M} \vec{D}^{2}\right) \psi
$$

where $c$ gives the measure of the interaction among particles mediated by the $U(1)$ gauge potential $a_{\mu}$, with the covariant derivatives given by $D_{t}=\partial_{t}+i q a_{0}, \vec{D}=\vec{\nabla}-i q \vec{a}$, and the anyon statistical parameter $\alpha$ to be identified as $\alpha \equiv q^{2} /(2 \pi c)$.

In the study of a quantum-mechanical ideal gas, the effect of the symmetry properties of the wave function can be interpreted, from a classical point of view, as a consequence of a fictitious classical potential introduced by Uhlenbeck and Gropper [8], referred to as effective statistical potential, which represents the first quantum correction for the classical $\mathrm{PF}$ [9, 10]. For our purposes we have to consider the two-body case, which is relevant for the subsequent computation of the statistical interparticle potential [9, 10]. The statistical potential completely determines the second virial coefficient, which gives the thermodynamical properties of the system in the dilute (high-temperature) regime. For the two-anyon system, after separating in (11) with $N=2$ the center-of-mass dynamics (i.e. that of a particle having mass $2 M$ ), one is left with the dynamics of the relative wavefunction:

$$
-\frac{1}{M}\left[\vec{\nabla}_{r}-i \alpha \vec{a}(\vec{r})\right]^{2} \psi(\vec{r})=E \psi(\vec{r})
$$

where $\vec{r}=\vec{r}_{1}-\vec{r}_{2}$ is the relative coordinate, and $a^{i}(\vec{r})=\epsilon^{i j} \frac{x^{j}}{r^{2}}$. Therefore the relative dynamics is equivalent to that of a single particle in presence of a point vortex $\vec{a}(\vec{r})$ placed at the origin.

An analysis of the statistical interparticle potential for an ideal gas of Abelian anyons is presented in [11, 12]. The eigenfunctions of (3) reads

$$
\Psi_{\alpha}=e^{i \mathbf{K} \cdot \mathbf{R}} e^{i l \theta} J_{|l-\alpha|}(k r) \equiv e^{i \mathbf{K} \cdot \mathbf{R}} \psi_{\alpha},
$$

where the capital (italic) letters respectively refer to center-of-mass (relative) coordinates. The bosonic description used in (11) imposes the condition $l=$ even; furthermore $J_{m}(x)$ denote the Bessel functions of the first kind [14] (their definition is recalled in Appendix A). Notice that the wavefunctions (44) are the eigenfunctions of (3) provided that the hard-core boundary conditions $\Psi_{\alpha}(0)=0$ are imposed. 
The two-body $\mathrm{PF}$ is $Z=\operatorname{Tr} e^{-\beta H_{2}}=2 A \lambda_{T}^{-2} Z^{\prime}$, where $Z^{\prime}$ is the single-particle $\mathrm{PF}$ in the relative coordinates, $\beta=1 / k_{B} T, \lambda_{T}=\left(\beta h^{2} / 2 \pi M\right)^{1 / 2}$ is the thermal wavelength and $A$ is the area of the system. The relative $\mathrm{PF} Z^{\prime}$ is given by

$$
Z^{\prime}=\frac{1}{h^{2}} \sum_{l=-\infty}^{\infty} \int d^{2} p \int d^{2} r e^{-\beta p^{2} / M}\left|\psi_{\alpha}\right|^{2}
$$

(with $p=\hbar k$ ). It is possible to conveniently rewrite Eq.(5) by using the following integral property [25] of the Bessel functions:

$$
\int_{0}^{\infty} e^{-\alpha x} J_{\nu}(2 \beta \sqrt{x}) J_{\nu}(2 \gamma \sqrt{x}) d x=\frac{1}{\alpha} I_{\nu}\left(\frac{2 \beta \gamma}{\alpha}\right) e^{-\left(\beta^{2}+\gamma^{2}\right) / \alpha},
$$

where $I_{\nu}$ is the modified Bessel function of the first kind [14] (see also Appendix [A). The relative PF then takes the form

$$
Z^{\prime}=\frac{1}{2} \sum_{n=-\infty}^{\infty} \int_{0}^{\infty} d x e^{-x} I_{|2 n-\alpha|}(x)
$$

where

$$
x \equiv \frac{M r^{2}}{2 \beta \hbar^{2}}=\frac{\pi r^{2}}{\lambda_{T}^{2}} .
$$

The one-body PF for classical systems, used in [8] to define the concept of effective statistical potential, is

$$
Z^{\prime}=\frac{1}{2 h^{2}} \int d^{2} p e^{-\beta p^{2} / M} \int d^{2} r e^{-\beta v(r)}:
$$

therefore, comparing (77) and (99) produces as a result [11]

$$
e^{-\beta v_{\alpha}(r)}=2 e^{-x} \sum_{n=-\infty}^{\infty} I_{|2 n-\alpha|}(x)
$$

(see Appendix B for details). Eq.(10) is plotted in Fig [1.

For the considerations which follow, it is convenient to introduce the function

$$
\mathcal{M}_{\alpha}(x) \equiv \sum_{n=-\infty}^{\infty} I_{|2 n-\alpha|}(x)
$$

so that the statistical potential can be written as

$$
e^{-\beta v_{\alpha}(r)}=2 e^{-x} \mathcal{M}_{\alpha}(x)
$$

The inter-particle statistical potential admits a closed expression in terms of the bivariate Lommel functions (alias, Lommel functions of two variables). Indeed, as evident from Appendix A, it is

$$
\mathcal{M}_{\alpha}(x)=i^{-\alpha} U_{\alpha}(i x, i x)-i^{\alpha} U_{2-\alpha}(i x, i x)
$$




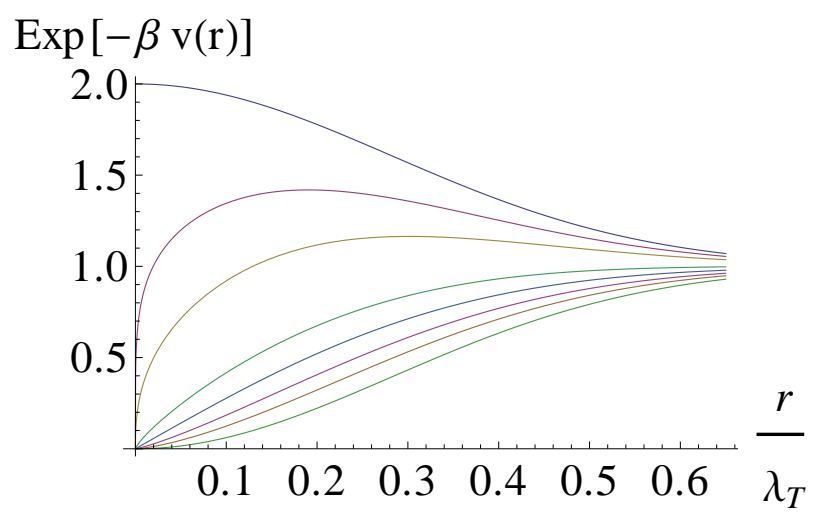

FIG. 1. Plot of $e^{-\beta v_{\alpha}(r)}$ vs. $r / \lambda_{T}$ for different values of the statistical parameter: from top to the bottom it is $\alpha=0,0.1,0.2,0.4,0.5,0.6,0.7,1$.

where $U_{\alpha}$ denote the Lommel functions of two variables [14]. Notice that the symmetry property $v_{\alpha}(r)=v_{2-\alpha}(r)(\forall \alpha \in \mathcal{R})$ holds for the statistical potential.

In Appendix $\mathrm{B}$ we prove the following integral representation for $v_{\alpha}(r)$, which will result useful in Subsection ஹA for discussing the large-distance limit behaviour:

$$
e^{-\beta v_{\alpha}(r)}=1+e^{-2 x} \cos \alpha \pi-2 \frac{\sin \alpha \pi}{\pi} e^{-x} \int_{0}^{\infty} d t \frac{\sinh [(1-\alpha) t]}{\sinh t} e^{-x \cosh t}
$$

Other integral representations for $e^{-\beta v_{\alpha}(r)}$ are presented in Appendix B. In the remaining parts of this Section we discuss the asymptotic behaviour of $v_{\alpha}$ for small and large values of the dimensionless parameter $x$, and we give a closed expression for the statistical potential $v_{\alpha}(r)$ in terms of the inverse Laplace transform of an algebraic function of $r$ and $\alpha$ : this manipulation allows us to straightforwardly regain the known expressions for $v_{\alpha}(r)$ in the bosonic/fermionic cases, to obtain its expression in the case of semions and to finally recover the value of the second virial coefficient for a generic $\alpha$ presented in [16].

\section{A. Limit behaviours}

In order to quantitatively understand the tendency of anyons to bunch together or vice versa to repel each other in different limit regimes of density, let's recall and further discuss the asymptotic behaviors of their effective statistical potential, for small and large distances [12, 13]. 
For small $r$ (that is $x \ll 1$ ) we can approximate the summation term in (10) as

$$
\begin{aligned}
\sum_{n=-\infty}^{\infty} I_{|2 n-\alpha|}(x) & \approx \sum_{n=0}^{\infty}\left[\frac{1}{\Gamma(2 n+\alpha+1)}(x / 2)^{2 n+\alpha}+\frac{1}{\Gamma(2 n+3-\alpha)}(x / 2)^{2 n+2-\alpha}\right] \approx \\
& \approx[\Gamma(\alpha+1)]^{-1}(x / 2)^{\alpha}+[\Gamma(3-\alpha)]^{-1}(x / 2)^{2-\alpha} .
\end{aligned}
$$

Since

$$
\beta v_{s}(r)=-\ln \left[2 e^{-x} \sum_{n=-\infty}^{\infty} I_{|2 n-\alpha|}(x)\right] \approx-\ln \left[2 e^{-x}\left([\Gamma(\alpha+1)]^{-1}(x / 2)^{\alpha}+[\Gamma(3-\alpha)]^{-1}(x / 2)^{2-\alpha}\right)\right]
$$

it follows

$$
\beta v_{\alpha}(r) \approx\left\{\begin{array}{lr}
-\ln \left[2-2 \pi r^{2} / \lambda_{T}^{2}\right] \approx-\ln 2+\frac{\pi}{2}\left(r / \lambda_{T}\right)^{2}, & \alpha=0,2 \\
-\ln \left[2\left(\pi r^{2} / 2 \lambda_{T}^{2}\right)^{\alpha} / \Gamma(\alpha+1)\right], & 0<\alpha<1 \\
-\ln \left[2 \pi r^{2} / \lambda_{T}^{2}\right], & \alpha=1 \\
-\ln \left[2\left(\pi r^{2} / 2 \lambda_{T}^{2}\right)^{2-\alpha} / \Gamma(3-\alpha)\right], & 1<\alpha<2
\end{array}\right.
$$

We may summarize the small distance behaviour as follows: $v_{\alpha}(r)$ is repulsive and logarithmically divergent to $+\infty$ for any $\alpha \in(0,2)$, whereas for $\alpha=0,2$ it is attractive, and quadratically increasing in $r$ starting from the finite value $v_{0}(r=0)=-\ln 2$. The small- $r$ asymptotic function for $v_{\alpha}(r)$ is discontinuous in $\alpha=1$, being twice than the limit asymptotic functions for $\alpha \rightarrow 1^{ \pm}$(in fact two equal Bessel terms contribute to the asymptotic behaviour for $\alpha=1$, whereas only one of them dominates when $\alpha \neq 1$ ).

To study the behavior of the statistical potential for large distance $r$ (i.e. $x \gg 1$ ) we employ the integral representation (14) of the modified Bessel function. The method of steepest descent allows to evaluate to an arbitrary order the last term of this integral representation. At the first significant order, we get

$$
-2 \frac{\sin \alpha \pi}{\pi} e^{-z} \int_{0}^{\infty} d t \frac{\sinh [(1-\alpha) t]}{\sinh t} e^{-z \cosh t} \approx \frac{2(\alpha-1) \sin \alpha \pi}{\sqrt{2 \pi z}} e^{-2 z} .
$$

Therefore the large distance behavior of the statistical potential is given by

$$
\beta v_{s}(r) \approx\left[-\cos \alpha \pi+\frac{\sqrt{2}(1-\alpha) \sin \alpha \pi}{\pi r / \lambda_{T}}\right] e^{-2 \pi r^{2} / \lambda_{T}^{2}}
$$

for any $\alpha \in[0,2]$. Let us notice that this result differs from the corresponding one in [12], and that the asymptotic behaviours for $\alpha=0, \frac{1}{2}, 1$ (see formulas (26), (27), (28) in the sequel) are correctly retrieved. The statistical potential for large distance is vanishing for 


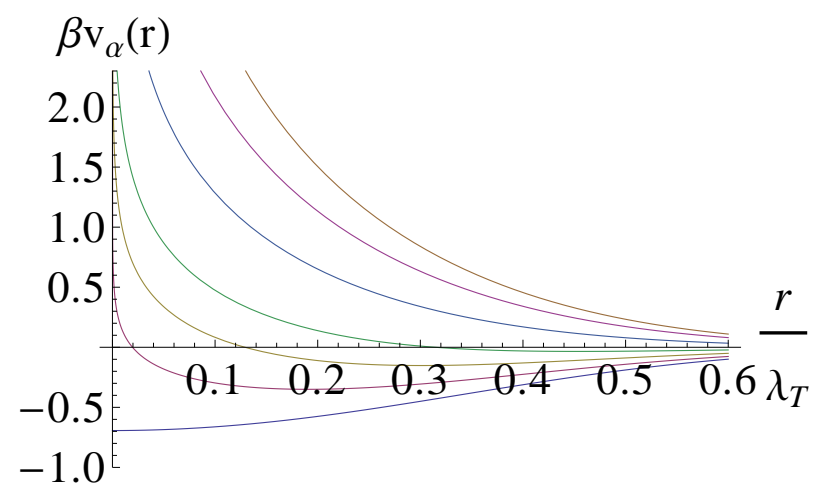

FIG. 2. $\beta v_{\alpha}(r)$ vs. $r / \lambda_{T}$ for different values of $\alpha$ : from top to bottom it is $\alpha=$ $1,0.7,0.5,0.3,0.2,0.1,0$. The bosonic curve $(\alpha=0)$ is monotonically increasing while the fermionic curve $(\alpha=1)$ is monotonically decreasing and divergent for $r \rightarrow 0$. All the curves for $0<\alpha<1 / 2$ diverge for $r \rightarrow 0$ and have a minimum point.

$r \rightarrow \infty$, and the interval $\alpha \in[0,1]$ (as well as the interval $\alpha \in[1,2]$, due to the symmetry property $\left.v_{\alpha}=v_{2-\alpha}\right)$ is divided in two regions: for large distance, $v_{\alpha}(r)$ is attractive for $0 \leq \alpha<1 / 2$, and repulsive for $1 / 2 \leq \alpha \leq 1$.

The large-distance and short-distance behaviors, considered together, imply that $v_{\alpha}(r)$ must admit a minimum point at finite distance, $r_{c r}(\alpha)$ for any $0<\alpha<1 / 2$ (see Fig,2). We denote the corresponding dimensionless quantity by $x_{c r}(\alpha) \equiv \pi r_{c r}^{2}(\alpha) / \lambda_{T}^{2}$. The minimum point $x_{c r}(\alpha)$ tends to $+\infty$ for $\alpha \rightarrow \frac{1}{2}^{-}$, as shown in Fig 3 ,

Fig 2 clearly shows that Abelian anyons have, from the point of view of the statistical potential, a "quasi-bosonic" behavior for $0<\alpha<1 / 2$ (i.e., $v_{\alpha}(r)$ is non-monotonic with a minimum) and a "quasi-fermionic" behavior for $1 / 2 \leq \alpha<1\left(v_{\alpha}(r)\right.$ is monotonically decreasing without a minimum).

\section{B. Laplace transform of the statistical potential and the $2^{\text {nd }}$-virial coefficient}

In this Section we write an explicit formula for $e^{-\beta v_{\alpha}(x)}$ as the inverse Laplace transform of a function of $x$ and $\alpha$. This result will allow us to write a simple formula for the statistical potential for the semionic gas and to compute the second virial coefficient, which of course coincides with the result reported in the seminal reference [16].

Let's start by writing down the Sumudu transform [15] of the function $\mathcal{M}_{\alpha}$, defined in 


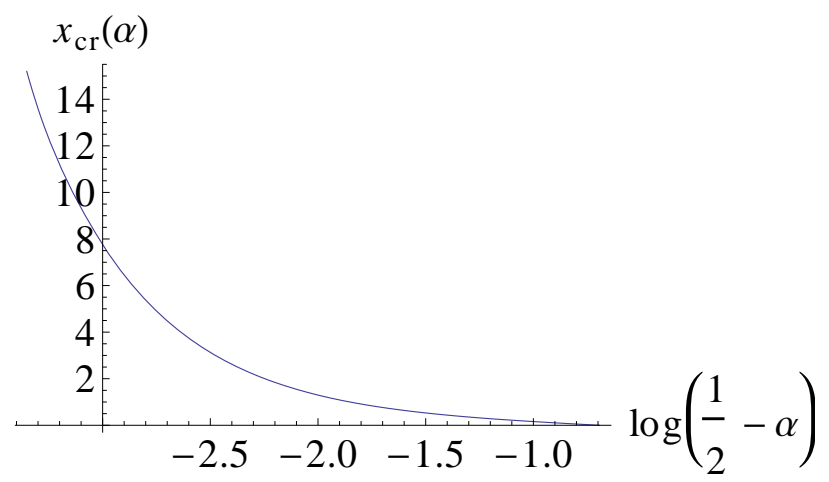

FIG. 3. The dimensionless value of the minimum point $x_{c r}(\alpha)$ of the statistical potential $v_{\alpha}$ of Abelian anyons as a function of the statistical parameter $\alpha$ near $\alpha=1 / 2$.

Eq.(11). The Sumudu transform of $\mathcal{M}_{\alpha}$ is defined as

$$
\left[\mathcal{M}_{\alpha}(x)\right]_{S} \equiv \int_{0}^{\infty} e^{-\theta} \mathcal{M}_{\alpha}(\theta x) d \theta=\int_{0}^{\infty} e^{-t / x} \mathcal{M}_{\alpha}(t) \frac{d t}{x}=s \int_{0}^{\infty} e^{-s t} \mathcal{M}_{\alpha}(t) d t
$$

where $s \equiv \frac{1}{x}$, whence

$$
\left[\mathcal{M}_{\alpha}\left(\frac{1}{x}\right)\right]_{S}=x \mathcal{L}\left[\mathcal{M}_{\alpha}\right](x)
$$

$\mathcal{L}$ being the ordinary (one-sided) Laplace transform. The function $\mathcal{M}_{\alpha}(x)$ is given by

$$
\mathcal{M}_{\alpha}(x)=\sum_{n=-\infty}^{\infty} I_{|2 n-\alpha|}(x)=e^{-\gamma \pi / 2 i} \sum_{n=0}^{\infty}(-1)^{n} J_{2 n+\gamma}(i x)+e^{-\alpha \pi / 2 i} \sum_{n=0}^{\infty}(-1)^{n} J_{2 n+\alpha}(i x)
$$

where $\gamma \equiv 2-\alpha$. Substituting (22) in (20), one gets that the Sumudu transform of $\mathcal{M}_{\alpha}$ becomes

$$
\left[\mathcal{M}_{\alpha}(x)\right]_{S}=e^{-\gamma \frac{\pi}{2} i} \sum_{n=0}^{\infty}(-1)^{n} \int_{0}^{\infty} e^{-t} J_{2 n+\gamma}(i x t) d t+e^{-\alpha \frac{\pi}{2} i} \sum_{n=0}^{\infty}(-1)^{n} \int_{0}^{\infty} e^{-t} J_{2 n+\alpha}(i x t) d t .
$$

The use of the integral properties of the Bessel functions of the first kind [14] (see pg. 386) gives the following expression:

$$
\begin{aligned}
{\left[\mathcal{M}_{\alpha}(x)\right]_{S} } & =\frac{e^{-\gamma \frac{\pi}{2} i}}{\sqrt{1-x^{2}}} \sum_{n=0}^{\infty}(-1)^{n}\left(\frac{\sqrt{1-x^{2}}-1}{i x}\right)^{2 n+\gamma}+\frac{e^{-\alpha \frac{\pi}{2} i}}{\sqrt{1-x^{2}}} \sum_{n=0}^{\infty}(-1)^{n}\left(\frac{\sqrt{1-x^{2}}-1}{i x}\right)^{2 n+\alpha}= \\
& =\frac{1}{\sqrt{1-x^{2}}}\left\{\left(\frac{\left(1-\sqrt{1-x^{2}}\right)}{x}\right)^{\gamma}+\left(\frac{\left(1-\sqrt{1-x^{2}}\right)}{x}\right)^{\alpha}\right\} \frac{1}{1-\left(\frac{1-\sqrt{1-x^{2}}}{x}\right)^{2}}= \\
& =\frac{1}{2}\left(\frac{1}{1-x^{2}}+\frac{1}{\sqrt{1-x^{2}}}\right)\left[\left(\frac{\left(1-\sqrt{1-x^{2}}\right)}{x}\right)^{\gamma}+\left(\frac{\left(1-\sqrt{1-x^{2}}\right)}{x}\right)^{\alpha}\right] .
\end{aligned}
$$


By sending $x \rightarrow 1 / x$ in (23), and applying (21), we obtain

$$
\mathcal{M}_{\alpha}\left(\frac{1}{x}\right)_{S}=\frac{1}{2}\left(\frac{x^{2}}{x^{2}-1}+\frac{x}{\sqrt{x^{2}-1}}\right)\left[\left(x-\sqrt{x^{2}-1}\right)^{\gamma}+\left(x-\sqrt{x^{2}-1}\right)^{\alpha}\right]
$$

and

$$
\mathcal{L}\left[\mathcal{M}_{\alpha}\right](x)=\frac{\left(x-\sqrt{x^{2}-1}\right)^{1-\alpha}+\left(x-\sqrt{x^{2}-1}\right)^{\alpha-1}}{2\left(x^{2}-1\right)} .
$$

Hence the inter-particle statistical potential admits the following form, for many purposes easier to handle than (10), since it does not contain infinite sums:

$$
e^{-\beta v_{\alpha}(r)}=e^{-x} \mathcal{L}^{-1}\left[\frac{\left(x-\sqrt{x^{2}-1}\right)^{1-\alpha}+\left(x-\sqrt{x^{2}-1}\right)^{\alpha-1}}{x^{2}-1}\right]
$$

The correct potentials for the bosonic and fermionic cases are straightforwardly reproduced: using known results for the Laplace transforms [26], we get

$$
\begin{aligned}
& e^{-\beta v_{\alpha=0}(r)}=e^{-x} \mathcal{L}^{-1}\left[\frac{2 x}{x^{2}-1}\right]=e^{-x} 2 \cosh x=1+e^{-2 x} \\
& e^{-\beta v_{\alpha=1}(r)}=e^{-x} \mathcal{L}^{-1}\left[\frac{2}{x^{2}-1}\right]=e^{-x} 2 \sinh x=1-e^{-2 x}
\end{aligned}
$$

Eq.(25) gives a closed formula for the potential in the case of semions $(\alpha=1 / 2$, or $\alpha=3 / 2)$ :

$$
e^{-\beta v_{\text {sem. }}(r)}=e^{-x} \mathcal{L}^{-1}\left[\frac{\left(x-\sqrt{x^{2}-1}\right)^{1 / 2}+\left(x-\sqrt{x^{2}-1}\right)^{-1 / 2}}{x^{2}-1}\right]=\operatorname{erf}(\sqrt{2 x})
$$

where erf is the error function [27].

Eq.(25) allows us to recover the second virial coefficient of a gas made of identical Abelian $\alpha$-anyons, which is given by [16]:

$$
B_{2}(\alpha, T)=\frac{1}{4} \lambda_{T}^{2}\left(-1+4 \alpha-2 \alpha^{2}\right)
$$

The link between the $2^{\text {nd }}$-virial coefficient and the statistical potential can be expressed in the form

$$
B_{2}(\alpha, T)=\frac{\lambda_{T}^{2}}{2} \int_{0}^{\infty} d x\left[1-e^{-\beta v(x)}\right] .
$$

Using Eqs.(26)-(27)-(28), for the three special cases $\alpha=0,1$ and $1 / 2$ (corresponding respectively to bosons, fermions and semions) one immediately finds

$$
B_{2}(\alpha=0, T)=\frac{\lambda_{T}^{2}}{2} \int_{0}^{\infty} d x\left[1-\left(1+e^{-2 x}\right)\right]=-\frac{1}{4} \lambda_{T}^{2}
$$




$$
\begin{gathered}
B_{2}(\alpha=1, T)=\frac{\lambda_{T}^{2}}{2} \int_{0}^{\infty} d x\left[1-\left(1-e^{-2 x}\right)\right]=+\frac{1}{4} \lambda_{T}^{2} \\
B_{2}\left(\alpha=\frac{1}{2}, T\right)=\frac{\lambda_{T}^{2}}{2} \int_{0}^{\infty} d x[1-\operatorname{erf}(\sqrt{2 x})]=\frac{\lambda_{T}^{2}}{2} \int_{0}^{\infty} d y y[1-\operatorname{erf} y]=\frac{1}{8} \lambda_{T}^{2}
\end{gathered}
$$

as it should be.

Finally, the effective 2-body statistical potential written as in Eq. (25)allows us to easily recover the expression of the $2^{\text {nd }}$-virial coefficient even for a general statistical parameter $\alpha$. Indeed, by virtue of (25) and the dominated convergence theorem, one has

$$
\begin{gathered}
\frac{B_{2}(\alpha, T)}{\lambda_{T}^{2}}=\frac{1}{2} \int_{0}^{\infty} d x\left[1-e^{-\beta v(x)}\right] \\
=\frac{1}{2} \lim _{\varepsilon \rightarrow 0} \int_{0}^{\infty} d x\left[e^{-\varepsilon x}-e^{-(1+\varepsilon) x}\left(\mathcal{L}^{-1}\left[\frac{\left(x-\sqrt{x^{2}-1}\right)^{1-\alpha}}{x^{2}-1}\right]-\mathcal{L}^{-1}\left[\frac{\left(x-\sqrt{x^{2}-1}\right)^{\alpha-1}}{x^{2}-1}\right]\right)\right] \\
=\frac{1}{2} \lim _{\varepsilon \rightarrow 0}\left[\frac{1}{\varepsilon}-\mathcal{L}_{\mid x=1+\varepsilon}\left(\mathcal{L}^{-1}\left[\frac{\left(x-\sqrt{x^{2}-1}\right)^{1-\alpha}}{x^{2}-1}\right]-\mathcal{L}^{-1}\left[\frac{\left(x-\sqrt{x^{2}-1}\right)^{\alpha-1}}{x^{2}-1}\right]\right)\right] \\
=\frac{1}{2} \lim _{\varepsilon \rightarrow 0}\left[\frac{1}{\varepsilon}-\frac{\left(1+\varepsilon-\sqrt{2 \varepsilon+\varepsilon^{2}}\right)^{1-\alpha}+\left(1+\varepsilon-\sqrt{2 \varepsilon+\varepsilon^{2}}\right)^{\alpha-1}}{2 \varepsilon+\varepsilon^{2}}\right]=\frac{1}{4}\left(-1+4 \alpha-2 \alpha^{2}\right),
\end{gathered}
$$

that is just (29).

As a byproduct of Eqs. (12), (13), (30), (34), we find an interesting integral property relevant to the bivariate Lommel functions (new, at the best of our knowledge):

$$
\int_{0}^{\infty} d x\left\{1-2 e^{-x}\left[i^{-\alpha} U_{\alpha}(i x, i x)-i^{\alpha} U_{2-\alpha}(i x, i x)\right]\right\}=-\frac{1}{2}+2 \alpha-\alpha^{2} .
$$

\section{STATISTICAL POTENTIAL FOR NON-ABELIAN ANYONS}

In this Section we discuss the statistical interparticle potential for a two-dimensional system of $S U(2)$ NACS spinless particles. The NACS particles are pointlike sources mutually interacting via a non-Abelian gauge field attached to them [28]. As a consequence of their interaction, equivalent to a non-Abelian statistical interaction for a system of bosons, they are endowed with fractional spins and obey braid statistics as non-Abelian anyons.

Let's briefly introduce the NACS quantum mechanics [30 34]. The Hamiltonian describing the dynamics of the $N$-body system of free NACS particles can be derived by a 
Lagrangian with a Chern-Simons term and a matter field coupled with the Chern-Simons gauge term [34]: the resulting Hamiltonian reads

$$
H_{N}=-\sum_{i=1}^{N} \frac{1}{M_{i}}\left(\nabla_{\bar{z}_{i}} \nabla_{z_{i}}+\nabla_{z_{i}} \nabla_{\bar{z}_{i}}\right)
$$

where $M_{i}$ is the mass of the $i$-th particles, $\nabla_{\bar{z}_{i}}=\frac{\partial}{\partial \bar{z}_{i}}$ and

$$
\nabla_{z_{i}}=\frac{\partial}{\partial z_{i}}+\frac{1}{2 \pi \kappa} \sum_{j \neq i} \hat{Q}_{i}^{a} \hat{Q}_{j}^{a} \frac{1}{z_{i}-z_{j}} .
$$

In formula (36) $i=1, \ldots, N$ labels the particles, $\left(x_{i}, y_{i}\right)=\left(z_{i}+\bar{z}_{i},-i\left(z_{i}-\bar{z}_{i}\right)\right) / 2$ are their spatial coordinates, and $\hat{Q}^{a}{ }^{\prime}$ s are the isovector operators which can be represented by some generators $T_{l}^{a}$ in a representation of isospin $l$ [32]. The quantum number $l$ labels the irreducible representations of the group of the rotations induced by the coupling of the NACS particle matter field with the non-Abelian gauge field: as a consequence, the values of $l$ are of course quantized and vary over all the integer and the half-integer numbers, with $l=1 / 2$ being the smallest possible non-trivial value $(l=0$ corresponds to a system of free bosons). As usual, a basis of isospin eigenstates can be labeled by $l$ and the magnetic quantum number $m$ (varying in the range $-l,-l+1, \cdots, l-1, l$ ).

Hence the statistical potential depends in general on the value of the isospin quantum number $l$ and on the coupling $\kappa$ (and of course on the distance $r$ and the temperature $T)$. The quantity $\kappa$ present in the covariant derivative is a parameter of the theory. The condition $4 \pi \kappa=$ integer has to be satisfied for consistency reasons [29, 30]. In the following we denote for simplicity by $k$ the integer $4 \pi \kappa$.

For non-Abelian anyons, in analogy with (9), the effective statistical potential can be related to the relative PF according to the following expression:

$Z_{2}^{\prime}(\kappa, l, T)-Z_{2}^{\prime(n . i .)}(l, T)=\frac{1}{2 h^{2}} \int d^{2} p e^{-\beta p^{2} / M} \int d^{2} r\left[\exp [-\beta v(\kappa, l, r)]-\exp \left[-\beta v^{(n . i .)}(l, r)\right]\right]$,

where $v^{(n . i .)}(l, r)$ refers to the system of particles with isospin $l$ and without statistical interaction $(\kappa \rightarrow \infty)$. The potential $v^{(n . i)}(l, r)$ can be expressed in terms of the potentials $v_{\alpha=0}(r)$ and $v_{\alpha=1}(r)$ for the free Bose and Fermi systems (endowed with the chosen wavefunction boundary conditions). $Z_{2}^{\prime}(\kappa, l, T)-Z_{2}^{\prime(n . i .)}(l, T)$ is the (convergent) variation of the divergent PF for the two-body relative Hamiltonian, between the interacting case in exam and the non-interacting limit $\kappa \rightarrow \infty$. 
For hard-core boundary conditions on the relative two-anyonic vectorial wave-function, the quantity $v^{(n . i .)}(l, r)$ which enters Eq.(37) is given by the projection onto the bosonic/fermionic basis:

$$
e^{-\beta v^{(n . i .)}(l, r)}=\frac{1}{(2 l+1)^{2}} \sum_{j=0}^{2 l}(2 j+1)\left[\frac{1+(-1)^{j+2 l}}{2} e^{-\beta v_{\alpha=0}(r)}+\frac{1-(-1)^{j+2 l}}{2} e^{-\beta v_{\alpha=1}(r)}\right]
$$

in analogy with the procedure shown in [18 20] for the computation of the $2^{\text {nd }}$-virial coefficient. By using the results $e^{-\beta v_{\alpha}=0}(r)=1+e^{-2 x}, e^{-\beta v_{\alpha=1}(r)}=1-e^{-2 x}$, one then obtains

$$
\exp \left[-\beta v^{(n . i .)}(l, r)\right]=1+\frac{e^{-2 x}}{2 l+1}
$$

Notice that this non-interacting quantity exactly corresponds to $(-1)^{2 l}$ times the statistical potential for a system of identical $(2 l)$-spin ordinary particles (fulfilling the spin-statistics constraint) at the same temperature, similarly to what argued in [20] about the $2^{\text {nd }}$-virial coefficient for the same system.

In the interacting case (i.e. finite $k$ ), we can express the statistical potential in terms of statistical potentials of Abelian anyons:

$$
e^{-\beta v(k, l, r)}=\frac{1}{(2 l+1)^{2}} \sum_{j=0}^{2 l}(2 j+1)\left[\frac{1+(-1)^{j+2 l}}{2} e^{-\beta v_{\omega_{j}}^{B}(r)}+\frac{1-(-1)^{j+2 l}}{2} e^{-\beta v_{\omega_{j}}^{F}(r)}\right],
$$

where $\omega_{j} \equiv[j(j+1)-2 l(l+1)] / k$, and $v_{\omega_{j}}^{B}(r), v_{\omega_{j}}^{F}(r)$ are the potentials for the Abelian $\omega_{j}$-anyon gases respectively in the bosonic and fermionic bases, given by

$$
e^{-\beta v_{\omega_{j}}^{B}(r)}=2 e^{-x} \mathcal{M}_{\omega_{j}}(x)
$$

and

$$
e^{-\beta v_{\omega_{j}}^{F}(r)}=2 e^{-x} \mathcal{M}_{\omega_{j}+1}(x)
$$

Both (41) and (42) are periodic quantities under the shift $\omega_{j} \rightarrow \omega_{j}+2$; it follows

$$
e^{-\beta v(k, l, r, T)}=\frac{2 e^{-x}}{(2 l+1)^{2}} \sum_{j=0}^{2 l}(2 j+1)\left[\frac{1+(-1)^{j+2 l}}{2} \mathcal{M}_{\omega_{j}}(x)+\frac{1-(-1)^{j+2 l}}{2} \mathcal{M}_{\omega_{j}+1}(x)\right]
$$

Eq.(43) gives the statistical potential for a gas of NACS particles. 


\section{A. $2^{n d}$-virial coefficient}

An useful application (and check, at the same time) of Eq. (43) consists in computing the second virial coefficient. The analogous of (30) reads

$$
B_{2}(k, l, T)=\frac{\lambda_{T}^{2}}{2} \int_{0}^{\infty} d x\left[1-e^{-\beta v(k, l, r)}\right] .
$$

Substituting in its integrand both Eq.(43) and the following decomposition of the unity

$$
1=\frac{1}{(2 l+1)^{2}} \sum_{j=0}^{2 l}(2 j+1)\left[\frac{1+(-1)^{j+2 l}}{2}+\frac{1-(-1)^{j+2 l}}{2}\right]
$$

one obtains for $B_{2}(k, l, T)$

$\frac{\lambda_{T}^{2}}{2(2 l+1)^{2}} \sum_{j=0}^{2 l}(2 j+1) \int_{0}^{\infty} d x\left[\frac{1+(-1)^{j+2 l}}{2}\left(1-2 e^{-x} \mathcal{M}_{\omega_{j}}(x)\right)+\frac{1-(-1)^{j+2 l}}{2}\left(1-2 e^{-x} \mathcal{M}_{\omega_{j}+1}(x)\right)\right]$.

By virtue of (35) one has then

$B_{2}(k, l, T)=\frac{\lambda_{T}^{2}}{(2 l+1)^{2}} \sum_{j=0}^{2 l}(2 j+1)\left[\frac{1+(-1)^{j+2 l}}{2}\left(-\frac{1}{4}+\gamma_{j}-\frac{1}{2} \gamma_{j}^{2}\right)+\frac{1-(-1)^{j+2 l}}{2}\left(-\frac{1}{4}+\eta_{j}-\frac{1}{2} \eta_{j}^{2}\right)\right]$

where $\gamma_{j} \equiv \omega_{j} \bmod 2$ and $\eta_{j} \equiv\left(\omega_{j}+1\right) \bmod 2$. This result matches with previous results reported in literature [19, 20], see in particular Eqs.(38) and (41) of [20].

\section{B. Minimum points}

Using Eq.(43), we can study if the gas of NACS has a "bosonic-like", "quasi-bosonic" or "quasi-fermionic" behaviour, according to its characterization in terms of the statistical potential. In correspondence with the analysis carried out in Subsection ПA, we can address the problem of determining which points of the discrete parameter space $\{k, l\}$ are associated to the presence of an (interior) minimum point $r_{c r i t}(k, l, T)$ for the statistical potential $v(k, l, r, T)$ (which will be referred to as "bosonic" ones), which points correspond to a monotonically increasing $v(k, l, r, T)$ ("bosonic-like" points) and which ones instead correspond to a $v(k, l, r, T)$ monotonically decreasing in $r$ (the "quasi-fermionc" ones). To this aim, let's exploit the limit behaviors of $\exp [-\beta v(k, l, r, T)]$ for small distance $(x \ll 1)$ and large distance $(x \gg 1)$, which straightforwardly arise from Eqs.(15) and (18). Notice that 
at $x=0$ one has

$$
\left.e^{-\beta v(k, l, r=0)}=\frac{1}{(2 l+1)^{2}} \sum_{j=0}^{2 l}(2 j+1)\left[\left(1+(-1)^{j+2 l}\right) \delta\left(\gamma_{j}, 0\right)+\left(1-(-1)^{j+2 l}\right) \delta\left(\gamma_{j}, 1\right)\right]\right],
$$

where $\delta$ denotes the Kronecker delta function and $\gamma_{j} \equiv \omega_{j} \bmod 2$. For large distance it is

$$
e^{-\beta v\left(k, l, r \gg \lambda_{T}\right)} \approx\left\{\begin{array}{lr}
1+\frac{e^{-2 x}}{(2 l+1)^{2}} s(j, k, l), & \text { if } s(j, k, l) \neq 0 \\
1-\frac{e^{-2 x}}{(2 l+1)^{2} \sqrt{2 \pi x}} t(j, k, l), & \text { otherwise. }
\end{array}\right.
$$

where

$$
s(j, k, l) \equiv \sum_{j=0}^{2 l}(2 j+1)(-1)^{j+2 l} \cos \left(\omega_{j} \pi\right)
$$

and

$$
\left.t(j, k, l) \equiv \sum_{j=0}^{2 l}(2 j+1) \sin \left(\gamma_{j} \pi\right)\left[\left(1+(-1)^{j+2 l}\right)\left(\gamma_{j}-1\right)-\left(1-(-1)^{j+2 l}\right)\left(\eta_{j}-1\right)\right]\right]
$$

with $\gamma_{j} \equiv \omega_{j} \bmod 2, \eta_{j} \equiv\left(\omega_{j}+1\right) \bmod 2$.

Our results can be summarized in the "phase diagram" shown in Fig, 4 , in which we distinguish pairs of parameters $(k, l)$ for which $v(k, l, r, T)$ has an interior minimum point (in black), pairs for which the statistical potential is monotonically increasing in $r$ (in magenta), and the remaining pairs (which are left blank). In this way the black points denote a "quasibosonic" behaviour and the magenta ones denote a "bosonic-like" behaviour, according to the classification operated in Section II to extract information from the statistical potential for Abelian anyons: one sees that for non-Abelian anyons one has mixed regions in which quasi-bosonic and quasi-fermionic behavior alternate, separated by regions dominated by a quasi-bosonic behavior. Bosonic-like behaviour occurs only for pairs $(k, l)$ having one of the forms: $(k$ generic, $l=0),(k=1, l$ integer $)$, or $(k=2, l$ even $)$.

\section{CONCLUSIONS}

In this paper we have studied the two-body effective statistical potential (which models, in the dilute regime, the dominant term of the statistical interaction between the particles) of ideal systems of Abelian and non-Abelian anyons, described within the picture of flux-charge composites. In both cases we have derived closed expression for the statistical potential (in terms of known special functions) and we have studied its behavior. Asymptotic expansions 


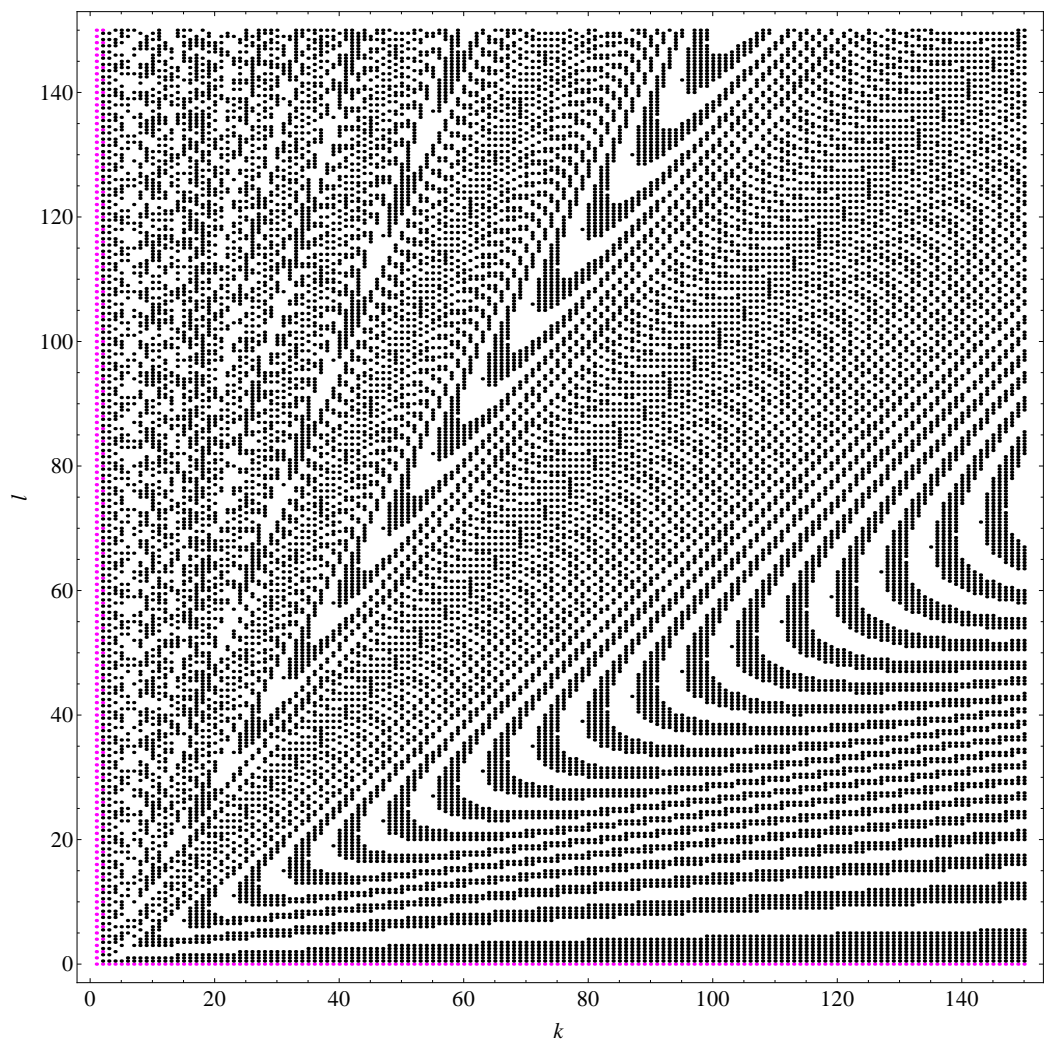

FIG. 4. Phase diagram in the parameter space $\{k, l\}$ for the family of non-Abelian anyon models under consideration. Points $(k, l)$ in the black regions correspond to the presence of an interior minimum point for the statistical potential $v(k, l, r, T)$ and are associated to quasi-bosonic behavior. Points in magenta correspond to a bosonic-like, monotonically increasing, behavior of the statistical potential. Finally, the remaining points are left blank and correspond to quasi-fermionic behavior.

have been provided, and the second virial coefficients of both systems have been found using a compact expression of the statistical potential in terms of Laplace transforms. A phase diagram for the non-Abelian gas as a function of the Chern-Simons coupling and the isospin quantum number has been derived.

In our study we have considered hard-core boundary conditions for the relative anyonic wave-functions: however, it would be interesting to use the results obtained so far in order to analyze the statistical potential in the more general soft-core conditions (both for the Abelian and non-Abelian ideal anyon gases).

Acknowledgements: We wish to thank Andrea De Luca and Gianfausto Dell'Antonio for very valuable suggestions and discussions. 


\section{Appendix A: Definition of the used Bessel functions}

In the main text we used the Bessel functions of the first kind $J_{\alpha}$ and the modified Bessel function of the first kind $I_{\alpha}$ : their definition is respectively given by

$$
J_{\alpha}(x)=\sum_{m=0}^{\infty} \frac{(-1)^{m}}{m ! \Gamma(m+\alpha+1)}\left(\frac{x}{2}\right)^{2 m+\alpha}
$$

and

$$
I_{\alpha}(x)=i^{-\alpha} J_{\alpha}(i x)=\sum_{m=0}^{\infty} \frac{1}{m ! \Gamma(m+\alpha+1)}\left(\frac{x}{2}\right)^{2 m+\alpha} .
$$

The Lommel functions of two variables are defined in Eq.(5) of pg. 537 of [14] and read

$$
\begin{gathered}
U_{\nu}(w, z)=\sum_{m=0}^{\infty}(-1)^{m}\left(\frac{w}{z}\right)^{\nu+2 m} J_{\nu+2 m}(z) \\
V_{n}(w, z)=\cos \left(\frac{w}{2}+\frac{z^{2}}{2 w}+\frac{\nu \pi}{2}\right)+U_{2-\nu}(w, z) .
\end{gathered}
$$

\section{Appendix B: Properties of the statistical potential $v_{\alpha}$}

In this Appendix we provide details and further informations on the statistical potential $v_{\alpha}$ for Abelian anyons.

We first give a derivation of Eq.(10): the relative PF (17) is given by

$$
Z^{\prime}=\frac{1}{2} \sum_{l=-\infty}^{\infty} \int_{0}^{\infty} d x e^{-x} I_{|l-\alpha|}(x), \quad x=M r^{2} / 2 \beta \hbar^{2}
$$

and it can be rewritten as

$$
Z^{\prime}=\frac{1}{2 h^{2}} \int d^{2} p e^{-\beta p^{2} / M} \int d^{2} r \sum_{l=-\infty}^{\infty} 2 e^{-M r^{2} / 2 \beta \hbar^{2}} I_{|l-\alpha|}\left(\frac{M r^{2}}{2 \beta \hbar^{2}}\right),
$$

as pointed out in [11]. That allows for its comparison with the PF (91) for classical systems associated to a generic potential $v(r)$, whence the result (10).

We also observe that

$$
e^{-\beta v_{\alpha}(r)}=2 e^{-x}\left[i^{-\alpha} \sum_{n=0}^{\infty}(-1)^{n} J_{2 n+\alpha}(i x)+i^{\alpha-2} \sum_{n=0}^{\infty}(-1)^{n} J_{2 n+2-\alpha}(i x)\right] .
$$

The expression that follows here below is a possible closed form for the statistical potential, but at the cost of using an integral representation given in formula (7), pg. 652 of [35]. It 
stands for any complex number $\mu$ in the vertical strip $-1<\operatorname{Re} \mu<\alpha$ :

$$
\begin{gathered}
e^{-\beta v_{\alpha}(r)}=2 e^{-x}\left[i^{-\alpha} \sum_{n=0}^{\infty}(-1)^{n} J_{2 n+\alpha}(i x)+i^{\alpha-2} \sum_{n=0}^{\infty}(-1)^{n} J_{2 n+2-\alpha}(i x)\right]= \\
=2 e^{-x}\left\{i^{-\alpha}\left[\frac{1}{2} \int_{0}^{i x} J_{\mu}(i x-t) J_{\alpha-\mu-1}(t) d t\right]+i^{\alpha-2}\left[\frac{1}{2} \int_{0}^{i x} J_{\mu}(i x-t) J_{1-\alpha-\mu}(t) d t\right]\right\}= \\
=e^{-x}\left[i^{-\alpha} \int_{0}^{i x} J_{\mu}(i x-t) J_{\alpha-\mu-1}(t) d t-i^{\alpha} \int_{0}^{i x} J_{\mu}(i x-t) J_{1-\alpha-\mu}(t) d t\right] .
\end{gathered}
$$

A simpler integral representation, valid for $\alpha \in(0,2)$, can be produced by using the following property ([14], pg. 540) of the bivariate Lommel function $U$ :

$$
U_{\nu}(w, z)=\frac{w^{\nu}}{z^{\nu-1}} \int_{0}^{1} J_{\nu-1}(z t) \cos \left\{\frac{1}{2} w\left(1-t^{2}\right)\right\} t^{\nu} d t, \quad \operatorname{Re}(\nu)>0
$$

together with expressions (B3), (13) and (A1). The resulting integral representation is

$$
e^{-\beta v_{\alpha}(r)}=2 x e^{-x} \int_{0}^{1} \cosh \left[\frac{x}{2}\left(1-t^{2}\right)\right]\left(I_{\alpha-1}(x t) t^{\alpha}+I_{1-\alpha}(x t) t^{2-\alpha}\right) d t .
$$

In the final part of this Appendix we provide the derivation of the integral representation (14). To this end, we use the following representation [25] for the modified Bessel function of the first kind:

$$
I_{\nu}=\frac{1}{\pi} \int_{0}^{\pi} e^{z \cos \theta} \cos \nu \theta d \theta-\frac{\sin \nu \pi}{\pi} \int_{0}^{\infty} e^{-z \cosh t-\nu t} d t, \quad \arg |z| \leq \frac{\pi}{2}, \operatorname{Re} \nu>0 .
$$

Then the summation term in (10) is:

$$
\begin{gathered}
\sum_{n=-\infty}^{\infty} I_{|2 n-\alpha|}(z)=\sum_{n=0}^{\infty} I_{2 n+2-\alpha}(z)+\sum_{n=0}^{\infty} I_{2 n+\alpha}(z)= \\
=\frac{1}{\pi} \int_{0}^{\pi} d \phi e^{z \cos \phi}\left[\sum_{n=0}^{\infty} \cos (2 n+\alpha) \phi+\sum_{n=1}^{\infty} \cos (2 n-\alpha) \phi\right]-\frac{1}{\pi} \int_{0}^{\infty} d t e^{-z \cosh t} f(t, \alpha),
\end{gathered}
$$

where

$$
f(t, \alpha)=\sum_{n=0}^{\infty} e^{-(2 n+\alpha) t} \sin (2 n+\alpha) \pi+\sum_{n=1}^{\infty} e^{-(2 n-\alpha) t} \sin (2 n-\alpha) \pi=\sin \alpha \pi \frac{\sinh [(1-\alpha) t]}{\sinh t},
$$

for $t \neq 0$ and

$$
f(0, \alpha) \equiv \lim _{t \rightarrow 0^{ \pm}} f(t, \alpha)=(1-\alpha) \sin \alpha \pi
$$


The first addend of the last integral representation is

$$
\begin{gathered}
\frac{1}{\pi} \int_{0}^{\pi} d \phi e^{z \cos \phi}\left[\sum_{n=0}^{\infty} \cos (2 n+\alpha) \phi+\sum_{n=1}^{\infty} \cos (2 n-\alpha) \phi\right]=\frac{1}{\pi} \int_{0}^{\pi} d \phi e^{z \cos \phi}\left[\sum_{n=0}^{\infty} \cos (2 n+\alpha) \phi+\right. \\
\left.+\sum_{n=-\infty}^{-1} \cos (2 n+\alpha) \phi\right]=\frac{1}{\pi} \int_{0}^{\pi} d \phi e^{z \cos \phi} \sum_{n=-\infty}^{+\infty} \cos (2 n+\alpha) \phi=\frac{1}{\pi} \int_{0}^{\pi} d \phi e^{z \cos \phi} \operatorname{Re}\left[\sum_{n=-\infty}^{+\infty}\left(e^{i \alpha \phi} e^{2 i n \phi}\right)\right]= \\
=\frac{1}{\pi} \int_{0}^{\pi} d \phi e^{z \cos \phi} \operatorname{Re}\left[e^{i \alpha \phi} 2 \pi \frac{\delta(2 \phi)+\delta(2 \phi-2 \pi)}{2}\right]=\frac{1}{2}\left(e^{z}+e^{-z} \cos \alpha \pi\right)
\end{gathered}
$$

As a result of (10), (B7) and (B8), one has then

$$
e^{-\beta v_{\alpha}(r)}=1+e^{-2 z} \cos \alpha \pi-2 \frac{\sin \alpha \pi}{\pi} e^{-z} \int_{0}^{\infty} d t \frac{\sinh [(1-\alpha) t]}{\sinh t} e^{-z \cosh t} .
$$

By direct inspection this result, notwithstanding the hypothesis of validity for (B6), is valid also for $\alpha$ at the extremes of the interval [0,2], so that the derivation of (14) is completed.

[1] Leinaas J M and Myrheim J 1977 Nuovo Cim. B 371

[2] Wilczek F 1990 Fractional statistics and anyon superconductivity (Singapore: World Scientific Publishing)

[3] Lerda A 1992 Anyons: quantum mechanics of particles with fractional statistics (Berlin: Springer-Verlag)

[4] Khare A 2005 Fractional statistics and quantum theory, 2nd ed. (Singapore: World Scientific Publishing).

[5] Nayak C, Simon S H, Stern A, Freedman M, and Das Sarma S 2008 Rev. Mod. Phys. 801083

[6] Yoshioka D 2002 The quantum Hall effect (Berlin: Springer-Verlag).

[7] Jain J K 2007 Composite fermions (Cambridge: Cambridge University Press)

[8] Uhlenbeck G E and Gropper L 1932 Phys. Rev. 4179

[9] Pathria R K 1972 Statistical mechanics (London: Pergamon)

[10] Huang K 1987 Statistical Mechanics (New York: John Wiley \& Sons)

[11] Kahng B and Park K 1992 Phys. Rev. B 458158

[12] Huang W H 1995 Phys. Rev. B 5215090

[13] Huang W H 2007 arXiv:cond-mat/0701715v2

[14] Watson G N 1966 Theory of Bessel Functions, 2nd Ed. (Cambridge: University Press)

[15] Watugala G K 1993 Int. Journ. of Math. Education in Science and Technology 2435 
[16] Arovas D. P, Schrieffer J R, Wilczek F, and Zee A 1985 Nucl. Phys. B 251117

[17] Lo H K 1993 Phys. Rev. D 484999

[18] Lee T 1995 Phys. Rev. Lett. 744967

[19] Hagen C R 1996 Phys. Rev. Lett. 764086

[20] Mancarella F, Trombettoni A and Mussardo G 2012 arXiv:1204.6656

[21] Aharonov Y and Bohm D 1959 Phys. Rev. 115485

[22] Stern A 2008 Ann. of Phys. 323204

[23] Hagen C R 1985 Phys. Rev. D 31848

[24] Jackiw R and Pi S Y 1990 Phys. Rev. D 423500

[25] Gradshteyn I S and Ryzhik I M 2007 Table of Integrals, Series, and Products, 7th edition (Academic Press)

[26] Bateman H and Erdélyi A 1954 Tables of Integral Transforms (New York: McGraw Hill)

[27] Olver F W J, Lozier D W, Boisvert R F and Clark C W 2010 NIST Handbook of Mathematical Functions (Cambrdige University Press)

[28] Dunne G V 1998 hep-th/9902115 (lectures at 1998 Les Houches Summer School "Topological Aspects of Low Dimensional Systems")

[29] Witten E 1989 Commun. Math. Phys. 121351

[30] Guadagnini E, Martellini M and Mintchev M 1990 Nucl. Phys. B 336581

[31] Verlinde E 1991, in Modern Quantum Field Theory (Singapore: World Scientific)

[32] Lee T and Oh P 1993 Phys. Lett. B 319 497; 1994 Phys. Rev. Lett. 72 1141; 1994 Ann. Phys. $\mathbf{2 3 5}, 413$

[33] Kim W T and Lee C 1994 Phys. Rev. D 496829

[34] Bak D, Jackiw R and Pi S Y 1994 Phys. Rev. D 496778

[35] Prudnikov A P, Brychkov I A and Marichev O I 1986 Integrals and Series, Vol. II (New York: Gordon and Breach Science Publishers) 